Hulagway at katotohanan ng edukasyon sa bagong himtang: Pagsikhay sa mga kaso ng pagtigil sa pag-aaral sa panahon ng pandemya

\author{
Galit, Gilbert C. $\bowtie$ \\ Leyte Normal University, Philippines (ggalit@lnu.edu.ph)
}

Received: 5 November 2021

Revised: 29 November 2021

Available Online: 2 January 2022 DOI: 10.5861 /ijrse.2022.213

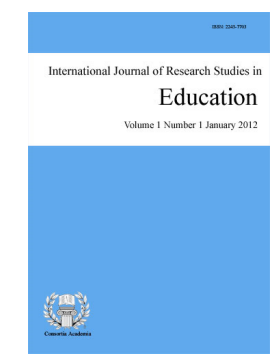

ISSN: $2243-7703$ Online ISSN: 2243-7711

OPEN ACCESS

\title{
Abstract
}

Education is considered to be one of the keys to uplift one's personal status and contribute to boost the national economy. However, due to Covid-19 outbreak, global system and mobility were affected, one of which is the education sector. Schools were closed and a new system in education has emerged - The New Normal, where classes are conducted online. With these, the desire to continue and graduate has become difficult for some. The transition of the educational system in many colleges and universities in the Philippines, from face-to-face to online has caused many problems to students. This study aimed to investigates the problems faced by students in online learning which caused them to drop-out from school. This is a qualitative research using case study design which seeks to illustrate realities in the current educational system. Using purposive sampling, participants were selected based on the researcher's designated criteria. A thematic analysis was employed for theming and coding the data obtained from the Focused Group Discussion (FGD). The investigation revealed that the lack of digital tools and resources, parental support, socioeconomic status, motivation to learn and their geographical location was a big problem for the students. These problems are some of the reasons for the cessation of their academic endeavor in times of pandemic. Consequently, research has strongly suggested to take into account the situation of students in the development and implementation of classroom and curricular policies in the new normal education.

Keywords: realities, new normal education, dropout case, academic cessation, pandemic 


\section{Hulagway at katotohanan ng edukasyon sa bagong himtang: Pagsikhay sa mga kaso ng pagtigil sa pag-aaral sa panahon ng pandemya}

\section{Introduction}

Ang edukasyon ay isa sa isinasaalang-alang bilang susi para pang-ekonomiya at panlipunang pag-unlad ng isang bansa. Isa itong mahalagang bahagi ng tagumpay ng isang indibiduwal at ng lipunan sa kabuoan. Ito ay proseso ng pagkuha, pagbabahagi at pag-iipon ng mga kaalaman. Tumutulong ito sa tao upang mapaunlad ang kanyang isipan, kakayahan, pang-unawa at pagkatao (Carlo, 2019). Subalit paano kung hindi lahat ng tao ay may kakayahan at pantay na access para sa isang magandang edukasyon? Sa Saligang Batas ng 1987, Artikulo XIV, Pangkat 1, sinasabing kinakailangang maitaguyod at maisulong ng Estado ang pantay na karapatan sa mga mamamayan para sa de-kalidad na edukasyon sa lahat ng antas, magsagawa ng mga naaangkop na hakbang upang gawing madali at accessible sa lahat ang nasabing edukasyon (officialgazette.gov.ph., nd).

Sa pagpasok ng taong 2020, hindi inaasahan ng lahat ang pagdating ng nakakamatay at nakakahawang "Corona Virus" na kilala sa tawag na "Covid 19". Naging sanhi ito ng pansamantalang paghinto ng mobilisasyong pandaigdig lalo na sa usaping ekonomiya. Isa sa mga lubhang naapektuhan ay ang sektor ng edukasyon na kung saan ay napilitang isara at ihinto na muna ang pagsasagawa ng tradisyonal na pagtuturo. Mahigit isang bilyong estudyante sa 186 na bansa ang naapektuhan dulot ng pandemiyang ito. Subalit parehong hindi nais ng lahat na ang mga mag-aaral ay matigil sa pag-aaral sa taong ito, ganoon din ang mga guro. Dahil dito, ang Department of Education (DepEd) ay naglabas ng opisyal na pahayag patungkol sa Learning Continuity Plan o ang "bagong normal" na pag-aaral na ipinakita sa mga magulang at mga mag-aaral noong ika-5 ng Mayo 2020. Samantala, ang Commission on High Education (CHED, 2020) ay naglabas din ng Memo Blg. 04, serye 2020, hinggil sa Guidelines and Implementation of Flexible Learning. Sa mga polisiyang ito, binigyang-pansin ang mga alternatibong paraan upang ipagpatuloy ang edukasyon sa new normal (Acuzar et al., 2021).

Ang mga hakbang na ito sa pagbabago ng sistemang pang-edukasyon ay sinang-ayunan ni Dhawan (2020), na sa panahon ng krisis, ang mainam na lunas ay ang pagpapatupad ng online learning mode. Dagdag pa nina Li at Lalani (2020) ng World Economic Forum, mas epektibo ang online learning kaysa sa pag-aaral sa loob ng silid paaralan sa panahong ito. Ang Online Learning Mode o Distance Learning ay maaaring gawin sa pamamagitan ng paggamit ng computer, smartphones at internet. Maaari rin naman ang paggamit ng modyular na kung saan may mga modyul (printed o electronic) na ipamamahagi sa mga estudyante na kanilang pag-aaralan at sasagutan sa itinakdang panahon (Calaor et al., 2020).

Matatandaan na noong ika-22 ng Mayo ay nagalabas ng pahayag si CHED chairman Prospero de Vera na kung saan binanggit niya na magiging bahagi na ng edukasyon ang flexible learning. Batay sa datos nina Acuzar et al. (2020), ang naging pahayag na ito ay nakalulungkot at nakagagalit para sa mga guro. Pinabulaanan ng pangkat ng mga guro ang pahayag na ito at inihayag na sa ganitong sistema ay disconnected sila sa mga mag-aaral na nasa ibaba. Dagdag pa, hindi lahat ay handa para rito, estudyante man o mga guro. Naniniwala rin ang mga guro na ang online learning ay hindi sapat upang matutunan ng estudyante ang mga life skill na kailangan.

Hindi handa ang gobyerno at kaguruan para sa transisyon mula Face-to-Face Learning patungo sa Online Learning kung kaya't labis na naapektuhan ang mga mag-aaral, Ayon kay Ang Probinsyano Party-List Rep. Ronnie Ong (binanggit ni Franche, 2020), hindi pa handa ang Department of Education (DepEd) para sa Online Learning, isinusulong niya ang pagkansela sa pagbubukas ng klase sa kasagsagan ng pandemya sapagkat aniya na hindi lahat ng mag-aaral ay kayang bumili ng gadgets at walang internet connection. Nangangamba si Ong na magiging mababa ang kalidad ng edukasyon dahil sa kawalan ng kahandaan sa online education. Ayon rin sa 
Hulagway at katotohanan ng edukasyon sa bagong himtang: Pagsikhay sa mga kaso ng pagtigil sa pag-aaral

pag-aaral ng School Division ng Batangas City (2020), nasa 50.40\% ang hindi handa sa technical readiness o access sa gadgets. Ang mga mag-aaral ay nasanay na mayroong nagtuturo o gumagabay sa kanilang pagkatuto nang ang pag-aaral ay ginagawa pa ng harapan o Face-to-face learning. Sa paglaganap ng Covid-19 ay nagkaroon ng bagong sistema ang edukasyon na Online Learning, sa sistemang ito walang gumagabay at napagtatanungan ang mga mag-aaral. Kung kaya't naging isang dagok ito sa mga mag-aaral.

Sa patuloy na pagpapatupad ng kasalukuyang sistema ng edukasyon, hindi lamang natuon ang suliranin sa kahandaan ng pagpapatupad nito. Higit na mas naging mabigat ito para sa mga mag-aaral na siyang dapat na bigyang-pansin dahil sila ang dapat na isaalang-alang sa ano mang programang pang-edukasyon ng pamahalaan. Naging malaking suliranin sa kasalukuyan ang pagbaba ng enrolment at paglobo ng bilang ng mga tumitigil sa pag-aaral.

Sa artikulo ni Gotinga (2020) inilahad niya ang datos na ipinakita ni Sen. Gatchalian na umabot sa 2.3 Milyon ang hindi nag-enrol sa taong panuruan 2020 nang mag-migrate ang sistema ng edukasyon mula face-to-face patungong distance learning. Batay din sa figyur na ipinakita ng DepEd, ang kabuuang enrolment sa mga pampubliko at pribadong paaralan, at sa state at mga lokal na unibersidad at kolehiyo sa pagitan ng taong 2020 at ang nakaraang taon ay bumaba ng halos $14 \%$ na katumbas ng 3.3 milyon na mas mataas pa sa bilang na nabanggit ni Gatchalian. Dagdag na niya, ang figyur na ito ay ipinapagpalagay na bilang ng dropouts sa kasalukuyan.

Ayon kay Ducanes at Tan (2020) mga ekonomista mula sa Ateneo de Manila University, ang ganitong pagtaas ng dropout rate ay hindi nakita sa halos ilang dekada. Ang matinding paglobo ng pagtigil sa pag-aaral ay kinakailangang matugunan sapagkat magdudulot ito ng pangmatagalang epekto sa kinabukasan ng mga mag-aaral. Binigyang-diin nila na ang mga tumigil sa taong ito sa pag-aaral ay malamang na magkaroon ng mababang tiyansa o pagkakataon na bumalik sa pag-aaral kahit pa matapos na ang pandemya. Dagdag pa ni Moore (2017), ang pagtigil sa pag-aaral ay hindi lamang nakaaapekto sa sariling buhay ng mga mag-aaral kung hindi higit sa lahat, sa ekonomiya ng bansa.

Karamihan sa mga mag-aaral ay pumapasok sa kolehiyo na may pag-asang mabigyan ang kanilang sarili ng pundasyon na kailangan nila upang maging matagumpay sa buhay o ang kasanayang kailangan nila upang makahanap ng magandang trabaho. Taon-taon, maraming bilang ng mga mag-aaral ang pumapasok sa kolehiyo, ngunit marami sa kanila ang madalas na nabibigo o tumitigil sa loob ng mas mababa sa tatlong taon. Ang pagtigil sa pag-aaral ay isang seryosong problema sapagkat inaalis nito sa bawat indibiduwal ang kanilang pangunahing karapatan sa edukasyon.

Sa datos ng CHED, umaabot sa 44, 069 na libo ang mga mag-aaral sa kolehiyo na hindi nagpatuloy sa taong panuruan 2020-2021. Karaniwang mga kadahilanan ay takot sa kontaminasyon, problemang pinansyal, kakulangan ng mga gadget, at ang kanilang lokasyon/tirahan na malayo sa mga paaralan (Magsambol, 2020). Sa pag-aaral nina Orion et al. (2014), nakita na ang pinakapangunahing dahilan ng pagtigil sa pag-aaral ng mga estudyante ang kakulangang pinansiyal. Para sa mga mag-aaral na mula sa mahirap na pamumuhay, tila mas malakas ang presyur ng pagtigil sa pag-aaral lalo pa kung tinitimbang ang pangangailangang pampamilya at ang mga gastusin sa paaralan.

Sa pag-aaral nina Ruhalahti et al. (2021) ipinakita na isa ang kondisyong pinansiyal sa mga salik na nakaaapekto sa pag-aaral ng mga estudyante sa kolehiyo sa panahon pandemya. Maliban dito, binigyang-diin ng pananaliksik ang kondisyon at kalagayan ng online learning. Sa kanilang papel, tinukoy ang limitadong pakikipagtulungan ng mga mag-aaral na resulta ng COVID-19 na maaaring magdulot ng negatibong damdamin, at ang paghiwalay (isolation) sa lipunan ay maaaring magpalitaw ng mga emosyong nauugnay sa stress. Dagdag $\mathrm{pa}$, ang independent learning ay maaaring maging madali lamang para sa mga estudyanteng matatalino samantalang maaaring maging napakabigat naman para sa ilan na nahihirapan sa pansariling pag-unawa at pagkatuto. Binigyang-diin pa ng mga mananaliksik ang kasaluyang krisis ay lalong nagpalala sa tinatawag na digital gap, hindi lahat ay may pantay na access sa mga kagamitang digital at may mabilis at magandang 
internet connection. Sa ganoon, ang mga nahihirapan sa ganitong sistema ay mas malaki ang posibilidad ng pagtigil sa pag-aaral sa kolehiyo.

Ang kalagayan ng edukasyon sa panahon ng pandemya ay mga hamon at oportunidad (del Castillo, 2021). Patunay dito ang mga nabanggit na pag-aaral na nagpapakita ng mga emperikal na datos sa tunay na kalagayan ng sistema. Lumitaw sa mga literatura at pag-aaral ang nakababahalang kaso sa pagtaas ng bilang ng pagtigil sa pag-aaral at ang ilan sa mga pangunahing dahilan nito. Sa kasalukuyang pananaliksik, binigyang-tuon ang pagsikhay sa mga danas ng mga mag-aaral sa pag-aaral sa bagong himtang (new normal). Nilayon nitong makita ang pinagmumulan ng mga mag-aaral upang higit na mas maunawaan ang kanilang kalagayan.

Ang datos ng pananaliksik ay magiging mainam na batayan para sa pagsasaayos ng mga patakarang pang-edukasyon partikular sa implementasyon ng distance learning. Makatutulong ito sa mga administrador ng mga paaralan upang maihanay ang mga angkop na polisiya upang matugunan ang pangangailangan ng mga estudyante at ganoon din matugunan ang suliranin sa pagtigil sap ag-aaral ng mga mag-aaral.

\subsection{Saligang Teoretikal}

Ginamit na saligan ng kasalukuyang pag-aaral ang mga teoryang Student Integration Model at Structural Strains Theory upang higit na mas maipaliwanag ang kaligiran ng pag-aaral lalo na sa pag-unawa sa mga dahilan kung bakit tumitigil ang mga mag-aaral sa pag-aaral. Sa pananaliksik na ito nakatuon ang pagsusuri sa konteksto ng edukasyon sa bagong himtang.

Ang Student Integration Model (SIM) ni Vicenti Tinto (1975) ay nakatuon sa tangkang maipaliwanag ang lahat ng aspeto at proseso na nakaiimpluwensya sa desisyon ng isang indibiduwal na lisanin ang pag-aaral sa kolehiyo o unibersidad, at kung ano ang ugnayan ng mga prosesong ito upang tuluyang tumigil sa pag-aaral. Nilayon ni Tinto sa teoryang ito na matukoy ang iba't ibang uri ng pag-uugali ng mga mag-aaral upang tumigil sa pag-aaral. Mahalaga ito sapagkat may iba't ibang dahilan na kung saaan ay maaaring piliin ng isang indibiduwal na umalis sa kolehiyo, o mapilitang tumigil sa pag-aaral (bartleby.com., 2021). Sa pag-aaral nina Willging at Johnson (2009), inilapat ang konsepto ng SIM sa pamamagitan ng pagpapalagay na ang pagtitiyaga ng isang indibiduwal ay nauugnay sa kung gaano kahusay ang pagganyak at kakayahang akademiko ng isang tao na umaayon sa mga katangiang pang-akademiko at panlipunan ng isang institusyon. Ang ugnayang ito ang humuhubog sa determinasyon ng isang tao na tapusin ang kolehiyo at/o pag-aaral sa isang institusyon.

Sa kasalukuyang pag-aaral, ang teorya ay inilapat upang maipakita ang mga dahilan ng pagtigil sa pag-aaral ng mga mag-aaral sa kolehiyo batay sa mga salik na may kaugnayan sa kanilang pag-uugali at pagtingin sa kalagayan ng edukasyon sa bagong himtang. Sa pamamagitan ng teoryang ito, ang pag-alam at pagsusuri sa mga salik na ito ay mabibigyang-linaw at makita ang tiyak na ugnayan ang suliraning kinaharap ng mga mag-aaral sa pag-aaral sa bagong himtang bilang pangunahing dahilan na nag-udyok sa kanila na tumigil sa pag-aaral.

Ganoon din, binigyang-tuon ng pananaliksik ang salik demograpikal ng mga mag-aaral upang higit na mas maunawaan ang dahilan ng pagtigil sa pag-aaral. Sa kontekstong ito, ginamit na saligan ang teoryang Structural Strains Theory ni Robert Merton (1938). Ayon sa K12academics.com. (2021), ang teorya na ito ay nakatuon sa ugnayan sa pagitan ng mga kadahilanan ng demograpiko, tulad ng katayuan sa socioeconomic, kasarian at etnisidad, at pag-dropout. Dagdag pa, ipinagpalagay ng nasabing teorya na ang demograpikong salik ng mga mag-aaral ay may malaking ambag sa mga kasong ito at karamihan sa mga tumitigil sa pag-aaral ay nagmumula sa mga pamilyang may mababang socioeconomic status.

Sa kasalukuyang pag-aaral ay tiningnan ng pananaliksik ang salik na ito bilang isang kontribyutor sa naging desisyon ng mga mag-aaral para tumigil. Ang mga suliraning kinaharap ng mga mag-aaral ay higit na mas naging mabigat dahil sa salik na ito gaya ng kakulangang pinansiyal, kalagayang heograpikal at kakulangan sa mga magagamit na materyal sa pag-aaral sa bagong himtang. 
Hulagway at katotohanan ng edukasyon sa bagong himtang: Pagsikhay sa mga kaso ng pagtigil sa pag-aaral

Ang mga nabanggit na teorya ay nagtalakay sa kaligirang suliranin ng pag-aaral. Pinagtitibay nito na ang desisyon ng isang indibiduwal na tumigil sa pag-aaral ay masisikhay sa pamamagitan ng pagsusuri sa aktuwal na kalagayan ng kanilang pagkatuo. Sa ganoon, ang mga mga pagpapasyang ito ay makikita sa mas malawak na saklaw gamit ang lente ng mga nabanggit na teorya. Sa ibaba ipinakita ang figyur ng pag-aaral bilang nagsilbing paradigma upang mabuo ang kasalukuyang pananaliksik.

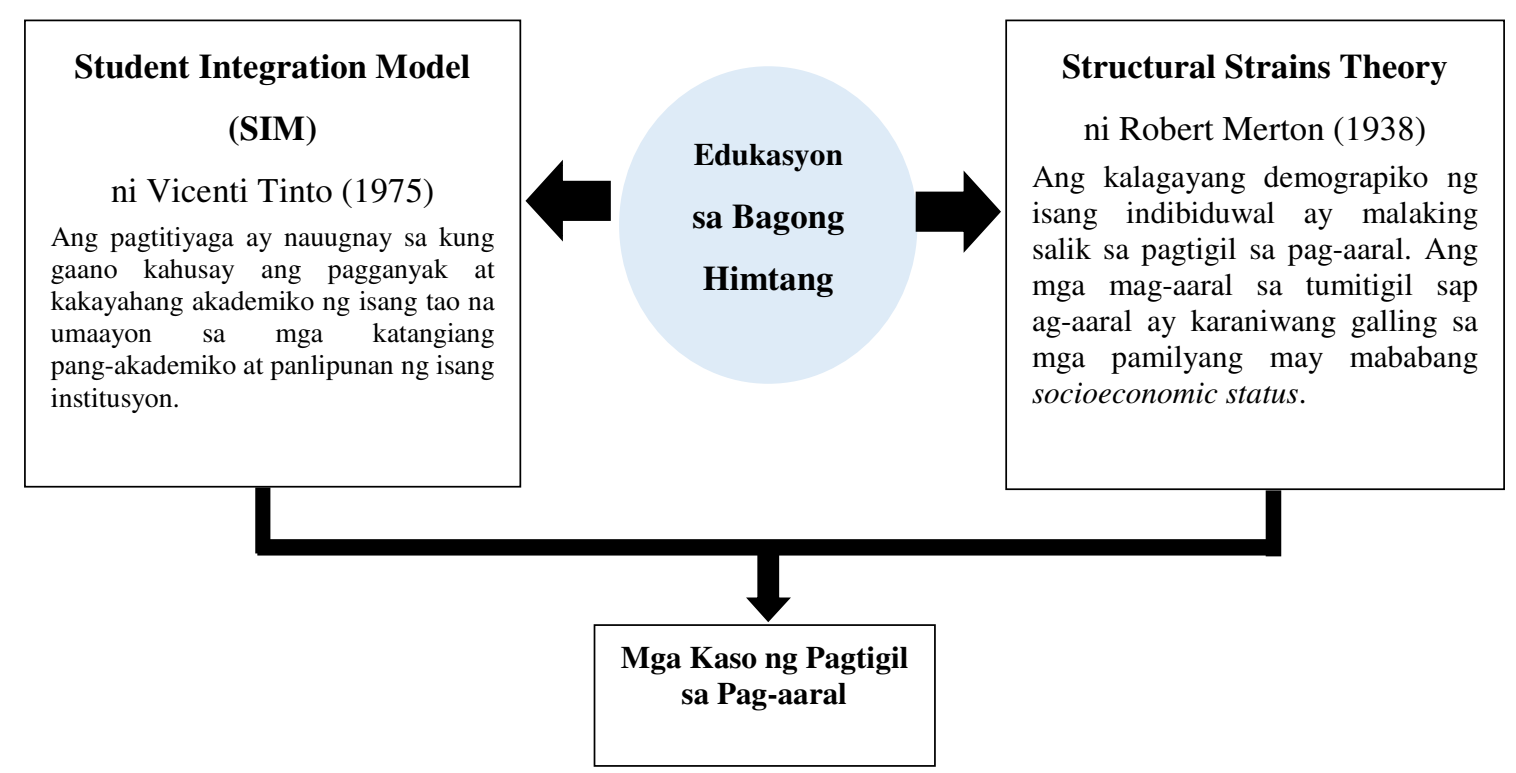

Figyur 1. Balangkas ng Pag-aaral

\subsection{Paglalahad ng Suliranin}

Pangunahing layunin ng kasalukuyang pag-aaral na maipakita ang larawan at katotohanan ng kalagayan ng edukasyon sa bagong himtang. Tinitiyak na pananaliksik na ito na masikhay ang mga kaso ng pagtigil sa pag-aaral sa pamamagitan ng pagsagot sa tanong na: (1) Ano ang mga suliraning kinaharap ng mga mag-aaral sa pag-aaral sa bagong himtang na nag-udyok sa kanila upang tumigil sa pag-aaral?

\section{Metodolohiya}

Ang pananaliksik na ito ay isang kuwalitatibong pag-aaral na ginamitan ng disenyong pag-aaral sa kaso (case study). Ayon kay McCombes (2019), ang pag-aaral sa kaso ay isang detalyadong pag-aaral ng isang tukoy na paksa, tulad ng isang tao, grupo, lugar, kaganapan, organisasyon, o hindi pangkaraniwang bagay. Ito ay mainam sa paglalarawan, paghahambing, pagsusuri at pag-unawa sa iba't ibang aspekto ng suliraning pampananaliksik. Binigyang-tuon ng pag-aaral ang pagsikhay sa mga kaso ng pagtigil sa pag-aaral sa edukasyon sa bagong himtang na ang tuon ay ang mga mag-aaral ng isa sa mga unibersidad sa Lungsod Tacloban. Gamit ang purposive sampling ay pinili ang mga kalahok na pagmumulan ng datos na pinili batay sa pamantayang binuo upang matiyak ang kalidad ng datos na makukuha. Una (1) ang kalahok ay dapat na mag-aaral sa kolehiyo, pangalawa (2) nakaranas ng e-learning mula sa taong panuruan 2020-2021 at pangatlo (3) tumigil sa pag-aaral sa panahon ng pandemya. Ang pangangalap ng datos ay isinagawa sa pamamagitan ng Focused Group Discussion (FGD) gamit ang semi-structured na talatanungan. Birtuwal ang pagsasagawa ng FGD upang maiwasan ang pisikal na kontak sa pagitan ng mananaliksik at mga kalahok upang maiwasan ang ano mang suliraning pangkalusugan. Limang (5) kalahok ang inimbatahan para sa pag-aaral subalit tatlo lamang (3) ang dumalo sa FGD. Ginamit din sa pananaliksik ang paraang pagtetema/pagkokoda bilang tritment sa mga datos na nakuha mula sa mga kalahok. Ang pagtetema/pagkokoda ay isang paraan kung paano tinutukoy ang datos na 
pinag-aaralan. Nagsasangkot ito ng pagkilala at pagtatala ng isa o higit pang mga sipi ng teksto o iba pang mga aytem sa datos tulad ng mga bahagi ng isang teksto na sa ilang mga kahulugan, ay nagpapakita ng pagkakatulad at parehong teoretikal at deskriptibong ideya (Gibbs, 2012). Ginamit ng mananaliksik ang paraang ito upang organisahin ang mga datos na nakuha mula sa isinagawang pagsusuri upang mabigyan ng angkop at tapat na interpretasyon. Muli ring ipinakita sa mga kalahok ang resulta ng panayam upang masigurado ang kapaniwalaan ng pagsusuri.

\section{Resulta at Diskusyon}

Sa bahaging matutunghayan ang resulta ng pag-aaral at pagtalakay sa mga datos na nakuha mula sa isinagawang panayam. Tinalakay sa bahaging ito ang mga suliraning kinaharap ng mga mag-aaral sa pag-aaral sa bagong himtang na nagbunsod ng pagtigil sa pag-aaral.

\subsection{Walang Signal at Mahinang Internet Connection}

Ang suliranin sa kawalan ng signal at mahinang internet connection ay naging suliranin sa pag-aaral ng mga mag-aaral sa edukasyon sa bagong himtang. Ang mga kalahok ay nakatira sa mga lugar na hindi abot ng signal at kung may signal naman, limitado at napakahina nito. Kinakailangan pa nilang pumunta sa mga katabing bayan na may signal para lamang makadalo sa kanilang mga klase. Dahil dito, naapektuhan ang kanilang akdemikong performans na naging sanhi upang tumigil na lamang sa pag-aaral. Ang sumusunod ay ang mga suliraning kinaharap ng mga mag-aaral dahil sa kawalan ng signal at mahinang internet conncetion.

Pakikipag-ugnayan sa mga Guro. Dahil sa kawalan ng access sa signal at internet. Ang mga mag-aaral ay hindi nakadadalo sa mga synchronous na pagkaklase. Dahil dito, nahirapan ang mga kalahok na maging updated at makasabay sa mga gawain at pagtalakay sa kanilang mga asignatura. Hindi rin makapagtanong ang mga kalahok sa kanilang mga guro kung sakaling may mga kinakailangang liwanagin sa nilalaman at mga panuto ng modyul. Ang kawalan ng komunikasyon ay nagbigay sa kanila ng negatibong emosyon sapagkat pakiramdam nila na mag-isa lamang nilang ginagawa ang pagkatuto. Ayon pa nga sa binanggit ni Vercide (2020) ang sistemang ito ay maaring makapagdulot sa mga mag-aaral ng 'feeling of isolation' o tila nakapuwesto sa isang lugar na kung saan hindi maaaring umalis o lumabas. Walang magaganap na maayos na pakikipag-usap o interaksyon sa mga kamag-aral kagaya ng isang normal na buhay estudyante.

Pagsusumite ng mga Awtput. Hindi maisumite ang mga pangangailangan sa mga asignatura sa oras na inilaan ng guro para sa pagpasa. Bagaman ayon sa mga kalahok na binibigyan naman sila ng pagkakataon ng kanilang mga guro na maisumite pa rin ang mga nahuling awtput, ang pagkahuli at ang pagtatambak at/o pagkaipon ng mga gawain ay naging suliranin pa rin sa kanila. Ang mga naiipong gawain na kailangang maipasa ay nakapagdulot ng hindi maggandang pagtingin sa pag-aaral. Bumaba ang kumpiyansa ng mga kalahok at nakaramdam ng pag-aalilangan sa kung magpapatuloy pa ba sa kanilang pag-aaral. Ayon sa mga kalahok, kapag may mga anunsiyo ang guro at hindi kaagad nababasa ay ipinaliliwanag naman nila ang kalagayan ng internet connection sa kanilang lugar. Mabuti na lamang at naiintindihan ito ng mga guro at nagbibigay ng pagkakataon na maipasa pa rin ang mga sagot/modyul. Ganoon pa man, dahil sa mahinang internet ay hindi pa rin naipapasa ang ibang modyul sa ibang asignatura na naging dahilan ng pagbaba ng kanilang performans dahil sa maraming kulang na awtput na hindi naipasa. Ayon pa sa pagbanggit ng isa sa mga kalahok:

"Nagdudurungan an mga modyul, sabay-sabay a pagpasa, nakukurian ak kay damo masydao ngan durungan. Dire ko tanan naipapasa, damo tak kulang. Inisip ko na amo manla gihap magdadamo ak INC ngan 3, gabay umundang la ngon-a."

["Nagsasabay-sabay ang mga modyul, sabay-sabay ang pagpasa, nahihirapan ako dahil masyadong marami at sabay-sabay pa. Hindi ko lahat naipapasa, marami akong kulang. Iniisip ko, ganoon din naman, dadami lang ang INC at 3 na marka, mas mabuting tumigil na muna.”] 
Hulagway at katotohanan ng edukasyon sa bagong himtang: Pagsikhay sa mga kaso ng pagtigil sa pag-aaral

Ang mga nabanggit na suliranin ay nauugnay sa pahayag ni Albay representative Joey Salceda (Binanggit ni Endraca, 2020), sa isang panayam sa radyo na kung saan sinabi niyang limang porsyento (5\%) lamang sa mga mag-aaral sa bansa ang may internet access na sapat upang magawa ng mga estudyante ang mga iaatas na gawain ng guro. Ayon pa kay ni Nieto (2020), malaki ang naging epekto ng pandemya sa mga guro at estudyante sa bansa. Nagdulot ito ng pangamba dahil sa posibleng pagbaba ng kalidad ng edukasyon. Nakapaloob dito ang pagsulong ng 'online classes' o ang paggamit ng internet at ng 'social networking sites' sa pag-aaral na ininda ng karamihan dahil sa kawalan ng kakayahan na magkaroon ng internet at bumili ng mga kagamitang panteknolohiya. Hindi lahat ng mga mag-aaral ay may laptop, cellphone, at internet na maaaring gamitin upang makapagpasa ng mga kinakailangan. Bagaman, ang implementasyon ng flexible learning ayon sa CMO. No. 4, s. 2020, na kailangang magbigay ng mga alternatibong paraan ang mga akademikong instituston gaya na lamang ng mga printed modules kung sakaling hindi sapat ang mga resources ng mga mag-aaral (CHED, 2020), hindi pa rin mapapasubalian ang suliraning kinakaharap ng mga mag-aaral sa edukasyon sa bagong himtang.

\subsection{Pag-unawa sa mga Aralin (Modyul)}

Sa edukasyon sa bagong himtang, ang pagkatuto ay nakasalalay sa magandang paghahanda at deliberi ng mga aralin. Sa flexible learning, gumagamit ang mga guro at mag-aaral ng modyul na maaaring printed o electronic. Ganoon pa man, ang pagkatuto ay kinakailangang kolaboratibo upang makamit ang mga inaasahang bunga ng isang kurso. Sa pananaliksik na ito, batay sa mga nalikom na datos ay nakita ang ilan sa mga suliraning kinaharap ng mga mag-aaral sa modyul na naging isa sa mga dahilan ng pagtigil sa pag-aaral.

Hindi sapat ang pagbabasa lamang. Isa sa mga naging pangunahing suliranin ng mga kalahok ay ang pag-unawa sa mga aralin na nakapaloob sa modyul. Ang modyul na ibinibigay ng guro ay karaniwang nangangailangan ng masinop na pagbabasa upang maunawaan ang paksa. Para sa mga kalahok, ang pagbabasa ay hindi nagiging sapat upang maunawaan ang nilalaman nito at maiugnay sa aktuwal at praktikal na senaryo. Dagdag pa, may mga sitwasyon sa modyul na masyadong teknikal at mahirap unawain. Dahil dito, hindi nagiging mabunga ang pagkatuto ng mga kalahok sa pag-aaral sa bagong himtang. Ang mga nilalaman ng modyul ay komplikado at minsan ay may napakahabang babasahin. Naging mahirap para sa mga kalahok ang pagkatuto at hindi nagiging sapat ang pagbabasa lamang upang masabing may natutunan sila sa aralin. Ang kakulangan sa pagbibigay ng mga halimbawa sa mga nilalaman ng modyul ay nakadaragdag sa suliranin sa pag-unawa ng mga kalahok. Ayon pa sa pagbanggit ng isa sa mga kalahok:

"Mahirap intindihin ang modyul, sana may mga halimbawa kung paano gagawin ang modyul.

Hindi ko ma-visualize ang iba dahil gusto ko ng halimbawa kung papano gagawin."

Kawalan $n g$ gabay. Ang kakulangan sa gabay sa pag-unawa sa modyul ay isa ring suliranin para sa mga kalahok. Bagaman isa sa mga kalahok ang nagsabing sinisikap niyang unawain ang paksa sa pamamagitan ng pagsasaliksik ay naging mahirap pa rin para sa kabuoan na maunawaan nang mabuti ang paksa. Ang paggabay ng mga guro ay napakahalaga upang maisakatuparan ang mga layunin ng aralin. Subalit sa kasalukuyang sistema, ang paggamit ng modyul (parehong printed at elektronik) ay nagsusulong ng tinatawag na independent learning na mahirap para sa ilang mag-aaral. Dagdag pa ng mga kalahok, may mga gawain sa modyul na kinakailangan ang paggabay ng kanilang guro upang magawa nang tama ang mga gawaing ito. Mas lalo itong naging mahirap para sa mga mag-aaral na ikinumpara pa ng isa sa mga kalahok na mas mahirap kaysa sa dating sistema nang ang pag-aaral ay isinasagawa pa ng harapan oface-to-face. Ayon pa sa pagbanggit ng isa sa mga kalahok:

"Mabigat ang mga gawain sa modyul lalo pa't walang guro na gumagabay. Sa face to face pa lamang mahirap na, ano pa sa online class."

Mabigat ang mga gawain. Ang mga kalahok ay may walong asignaturang kinukuha at bawat asignatura ay may modyul, at bawat modyul ay may 3 hanggang 4 na gawain. Pangunahing naging suliranin ng mga kalahok ang pag-unawa sa mga panuto sapagkat karamihan sa mga gawain sa modyul ay hindi malinaw at walang tiyak na hakbang kung papaano gagawin ang gawain. May mga pagkakataon ding pangkatan ang ibinibigay na gawain 
na ininda ng mga kalahok dahil nga sa kawalan ng komunikasyon sa kanilang mga kaklase. Dagdag pa, ang ilan sa mga gawain ay paggawa ng mga vidyo, vlog, report at iba na hindi kaya dahil sa limitadong resources ng mga kalahok.

Ang paggamit ng modyul (parehong printed at elektronik) bilang pangunahing kagamitan sa pagkatuto ay isa mga natatanging katangian ng edukasyon sa bagong himtang. Isinusulong nito ang independent learning na humahasa sa katangian ng pagiging responsable ng mga mag-aaral, at ang bigat ng mga gawain ay nag-uudyok ng mas mataas ng antas pag-iisip upang matuto (Nardo, 2017; Kuhl \& Eitel, 2016). Ganoon pa man, hindi pa rin mapapasubalianan na ang ganitong sistema ay may maraming pagsubok na kinakaharap at ilan lamang dito ang kawalan ng pisikal na interaksiyon sa pagitan ng mag-aaral at guro. Kaya naman, sa ganitong sistema ay walang ibang makatutulong sa mga mag-aaral kundi ang sariling sikap at istilo kung paano matututo (Vonderwell, 2003). Nauugnay ang mga suliranin ng mga kalahok sa pag-aaral nina Pe Dangle et al. (2020), lumabas na ang karamihan sa mga mag-aaral ay nahihirapan sa bagong sistema ng pag-aaral na ito. 90\% ng mga kalahok ay sinasabing nahirapan sila pagsagot sa kanilang mga modyul. Ang kalahati sa kanila ay walang sapat na oras upang magawa ang lahat ng kanilang mga modyul sa loob ng isang linggo. Madalas silang nakatatanggap ng hindi bababa sa 8 modyul sa lahat ng mga paksa at ang bawat modyul ay may 3-5 na gawain. Sa konteksto ng kasalukuyang pag-aaral, ang suliraning ito ay isa sa mga nakaapekto sa mga kalahok na tumigil sa pag-aaral.

\subsection{Kakulangan Pinansiyal}

Karaniwan ng dahilan ang kakulangang pinansiyal sa pagtigil sa pag-aaral ng mga mag-aaral sa kolehiyo. Gaya ng mga nabanggit sa pag-aaral nina Orion et al. (2014) at Ruhalahti et al. (2021) at ganoon din sa datos ng CHED (Magsambol, 2020) na isa ang pinansiyal sa mga salik na nakaiimpluwensiya sa desisyon ng pagtigil ng mga mag-aaral sa pag-aaral. Sa pananaliksik na ito, tinukoy ang ilang tiyak na suliranin kaugnay sa kakulangang pinansiyal ng mga kalahok.

Walang sapat na kagamitan. Ang mga kagamitan gaya ng cellphone at laptop ay isa sa mga naging suliranin ng mga kalahok. Walang sapat na kagamitan ang mga kalahok na magagamit sa pag-aaral online na naging dahilan kung bakit mas naging mahirap para sa kanila ang maipagpatuloy ang pag-aaral. Napakahalaga para sa mga kalahok ang kagamitang ito dahil dito nakasalalay ang lahat ng prosesong pampagkatuto sa kasalukuyang sistema. Ayon sa pagbanggit ng isa sa mga kalahok:

“Importante it laptop kay dida mo makukuha it ngatanan. Amo ito it imo pinakaklasrum."

["Napakahalaga ng laptop dahil dito mo makukuha ang lahat. Ito ang magsisilbing mong klasrum.”]

Sa kasalukuyang sistema na isinasagawa ang klase sa birtuwal na paraan (synchronous class), ang kawalan ng kagamitan gaya ng laptop ay katumbas ng kawalan ng pagkakataon na makadalo sa mga pagtalakay na isinasagawa sa iba't ibang online platforms. Marami rin sa mga gawain ay kinakailangang encoded na ipapasa sa google classroom ng klase. May cellphone man ang mga kalahok hindi ito kagaya ng mga high-tech model na maaaring makapag-download ng mga applications na magagamit at makatutulong sa pag-aaral. Ang ilan nga sa mga kalahok ay pinili ang printed modules para lang makapagpatuloy sa pag-aaral. Hindi gaya ng iba, ang kanilang mga sagot sa mga modyul ay isinusulat na lamang nila sa papel at kinukuhanan na lamang ng larawan para maisumite sa kanilang mga guro.

Kalagayang socioeconomic. Ang mga kalahok ay may mababang estadong socioeconomic na isa mga dahilan kung bakit hindi kayang makabili ng mga kagamitan para sa kanilang pag-aaral. Walang sapat na pinagkakakitaan, at walang permanenteng trabaho ang kanilang mga magulang. Ang load na isa sa mga pangangailangan upang makasabay sa mga gawaing online ay suliranin din sa mga kalahok. Sa pagsasagawa ng mga gawain sa modyul, kailangang mag-load ng kahit na 30 at 50 na tumatagal lamang ng tatlong (3) araw. 
Hulagway at katotohanan ng edukasyon sa bagong himtang: Pagsikhay sa mga kaso ng pagtigil sa pag-aaral

Dahil dito, hindi magawang maging aktibo ng mga kalahok sa pagdalo sa mga talakayang online dahil sa suliranin sa load. Isinaalang-alang ng mga kalahok ang kanilang kalagayan sapagkat para sa mga kalahok, may mas mahalagang pangangailangan sa kanilang bahay na dapat unahin kumpara sa pagbili ng load. Ayon pa nga sa pagbanggit ng isa sa mga kalahok:

"Hindi sapat ang kita ng magulang ko. Ginagawa naman nila ang paraan ngunit kulang pa dahil walang permanenteng trabaho at income ang aking mga magulang. Kahit CP nahihirapan ang mga magulang ko, tricycle driver at housewife ang magulang ko. broken family po kami."

Ang mga nabanggit na suliranin ay nauugnay sa pag-aaral nina Orion et al. (2014) na karaniwan sa mga mag-aaral na tumitigil sa pag-aaral ay mula sa mahirap na pamilya. Karamihan sa mga magulang ng mga dropouts ay walang permanenteng trabaho at kita at nagiging kultura na ng mga ganitong pamilya na sikaping mapag-aral ang mga anak upang maiahon sa hirap ang kanilang pamumuhay. Naging pangunahing hinuha sa kanilang pag-aaral na sa mga salik na polisiyang pampaaralan, ugnayang guro at mag-aaral, pinansiyal, akademikong performans at kurikulum - ang pinansiyal ang pinakapangunahing dahilan ng pagtigil ng mga mag-aaral sa pag-aaral. Ayon pa kay Gunigundo (2020), dapat alalahanin ng gobyerno ang iba't ibang pinanggagalingang estado sa buhay ng mga estudyante. Dito talaga makikita ang hindi pagkapantay-pantay sa mga mag-aaral kahit na sabihin na lahat ay pareho ang talakayan bawat baitang, ngunit ang mga kagamitan ay hindi pare-pareho. May mga mag-aaral na mayroon kaagad ng mga kagamitan, habang may mga mag-aaral na poproblemahin pa ng magulang kung saan kukuha ng pera para magka-internet o di kaya magrenta ng kompyuter para sa online classes. Dagdag na pasanin din kung saan kukuha ng pera para makapag-load.

\subsection{Suportang mga Magulang}

Mahalaga ang suporta ng mga magulang upang maging positibo ang mga mag-aaral sa kanilang pag-aaral nang hindi tumigil. Subalit sa kaso ng mga kalahok, naging mahirap lalo ang kanilang kalagayan dahil na rin sa hindi matibay ang pundasyon ng suporta ng kanilang mga magulang sa kanilang pag-aaral sa bagong himtang.

Karaniwang tinitingnan ng mga magulang ng mga kalahok ang pag-aaral sa bagong himtang (online learning) bilang isang paraan lamang upang makaiwas ang mga kalahok sa pagtulong sa mga gawaing bahay. Inaakala ng mga magulang na ginagawang dahilan lamang ng mga mag-aaral ang pagmo-module para iwasan ang ilang tungkuling pantahan. Iniisip din ng mga magulang na ang pagbababad sa kanilang mga cellphone ay pakikipag-chat at paglilibang lamang.

Maliban dito, ang pagtingin ng mga magulang ng mga kalahok sa online learning ay pag-aaksaya lamang ng oras. Dagdag pa, para sa mga magulang, gastos lamang ang online learning sapagkat siguradong hindi naman makapapasa ang mga kalahok dahil hindi naman makadadalo sa mga synchronous classes dulot nga ng kakulangan sa mga kagamitan na magagamit sa pag-aaral. Nakababahala rin ang pananaw ng mga magulang ng mga kalahok sa pagsasabing mainam kung magtrabaho na lamang ang kanilang mga anak upang makatulong sa pamilya. Ayon pa nga sa pagbanggit ng isa sa mga kalahok:

"Sabi ng mama ko, gastos lang 'yan, walang maidudulot na mabuti. Dapat daw magtrabaho na lamang ako para makatulong, nag-aaway ang mga magulang ko, nagagalit si mama kapag nagmomodyul ako. Naging hadlang ang mama ko nung nag-aaral ako."

Ikinababahala rin ng mga magulang ang labis na pagbababad ng mga anak sa mga gadgets gaya ng cellphone. Para sa kanila maaari itong makapagdulot ng hindi mabuti sa kalusugan ng kanilang mga anak. Dagdag pa, hindi pabor sa online learning ang isa sa mga mgulang ng mga kalahok sa paniniwalang kompromiso ang kalidad ng pagkatuto sa ganitong sistema ng pag-aaral. Ayon sa pagbanggit ng isa sa mga kalahok:

"It diploma pwede mo ito makuha, pero it kalidad hit hibaro dre basta-basta makukuha." 
[“Ang diploma ay maaaring makuha, pero ang kalidad ng pagkatuto ay hindi basta-bastang makukuha.”]

Dahil sa kakulangan ng suporta, nakararamdam ang mga kalahok ng pagsasarili at mabigat na pakiramdam na maipagpatuloy ang kanilang pag-aaral. Dahil dito, ang pagkatuto ay mas lalong nagiging mahirap sapagkat pakiramdam nila mag-isa lamang nila itong ginagawa at itinataguyod. Ang mga ganitong pakiramdam ay mas higit na nagdulot ng kalungkutan na nagbunsod ng negatibong epekto sa kanilang pag-aaral. Ayon pa nga sa pananaliksik nina Ruhalahti et al. (2021), ang kakulangan sa suporta mula sa kapwa, mga kaibigan at kaklase at ang di-magandang tanawing pang-akademiko ay nakaaapekto sa motibasyon ng isang mag-aaral.

Sang-ayon dito sina Ceka at Murati (2016) na nagsabing, kapag isinasangkot ng mga magulang ang kanilang sarili sa proseso ng edukasyon ng kanilang mga anak, kadalasan ang resulta ay maaaring maging kaaya-aya at positibong nakapagpapatibay sa pagpapahalaga ng isang mag-aaral. Kaugnay nito, sa pamamagitan ng kanilang pagpapakita ng suporta tungkol sa mga kakayahan ng kanilang mga anak at ang kanilang pangkalahatang kapasidad sa pagkatuto ay humahantong sa matagumpay na pag-aaral at sa edukasyon bilang isang kumplikadong proseso. Samakatuwid, ang mga magulang ay dapat makibahagi sa pagsuporta sa kanilang mga anak at magpakita ng natatanging suporta bilang isa sa mga pangunahing estratehiya na maghahatid ng matagumpay na edukasyon ng kanilang mga anak sa paaralan.

\section{Konklusyon at Rekomendasyon}

\subsection{Konklusyon}

Batay sa mga nakalap na datos, nabuo ang sumusunod na kongklusyon:

$>$ Ang kawalan ng signal at mahinang internet connection ang pinakapangunahing dahilan ng pagtigil ng mga mag-aaral sa pag-aaral sa bagong himtang. Nagdulot ito ng mga suliranin sa komunikasyon lalo na sa pakikipag-ugnayan sa guro na naging dahilan naman ng hindi pagiging updated at pagkahuli sa pagpasa ng mga awtput. Naging sanhi rin ito ng negatibong emosyon sa mga mag-aaral gaya ng kawalan ng kumpiyansa sa sarili na naging dahilan ng pagtigil sa pag-aaral.

$>\quad$ Ang mga suliranin sa pag-unawa sa mga nilalaman ng modyul at bigat ng mga gawain sa modyul ay isa ring dahilan ng pagtigil ng mga kalahok sa pag-aaral. Kailangan ng paggabay ng mga mag-aaral sa pag-unawa sa mga modyul at sa pagsasagawa ng mga gawaing nakapaloob dito.

$>$ Ang kakulangang pinansiyal ay isa ring kontribyutor sa pagtigil ng mga mag-aaral sa pag-aaral. Dahil sa salik na ito, walang sapat na mga kagamitan gaya ng cellphone, laptop at internet ang mga mag-aaral na magagamit sa pag-aaral. Mahalagang salik sa pag-aaral sa bagong himtang ang kalagayang socioeconomic ng mga mag-aaral.

$>$ Ang kawalan ng suporta mula sa mga magulang ay nagdulot ng hindi magandang epekto sa pag-aaral ng mga kalahok. Ang maling pananaw ng magulang hinggil sa online class ay naging isa sa mga dahilan ng pagtigil ng mga kalahok sa pag-aaral.

\subsection{Rekomendasyon}

Batay sa nabuong kongklusyon, inirekomenda ng pananaliksik na:

$>$ Sa pagpapatupad ng flexible learning, isaalang-alang ang lokasyon at kalagayang heograpikal ng mga mag-aaral. Magbigay ng mga alternatibo gaya ng paggamit ng mga printed modules at magtalaga ng mga pamantayang angkop sa sitwasyon ng mga mag-aaral.

D Balangkasin nang maayos ang mga modyul na ibinibigay sa mga mag-aaral. Gawing komprehensibo 
Hulagway at katotohanan ng edukasyon sa bagong himtang: Pagsikhay sa mga kaso ng pagtigil sa pag-aaral

ang pagtalakay at magbigay ng malinaw na panuto. Makatutulong ang pagdaragdag ng mga halimbawa. Iwasan din ang pagbibigay ng maraming gawain (tukuyin lamang ang mga pinakamahalagang kompetensi).

$>\quad$ Iwasan ang pagbibigay ng mga gawaing nangangailangan ng paggamit ng iba't ibang digital tools at digital resources gaya ng paggawa ng mga video, vlogs at iba pang katulad na gawain. Hanggat maaari iwasang dumepende ang komprehensibong pagtalakay sa mga paksa sa mga isinasagawang synchronous classes. Maiiwasan nito ang palagiang pagsasagawa ng talakayang online.

$>\quad$ Magsagawa ng oryentasyon (online) sa mga magulang upang maipaliwanag ang kaligiran ng online learning nang maiwasan ang maling pananaw tungkol dito. Talakayin din ang mahalagang papel na ginagampanan ng pamilya sa pag-aaral ng kanilang mga anak.

\section{Sanggunian}

Acuzar, R., Algar, E. \& Mercene, M. (2021). Kwentong distance learning: Mga tinig ng estudyante, magulang, at guro. Retrieved September 20, 2021 from https://rb.gy/6j8tbh

Batangascity.gov.ph. (2020). Survey sa kahandaan ng guro, estudyante at magulang sa distance learning isinagawa. Retrieved September 2021, from https://rb.gy/9zzzom

Bartleby.com. (2021). Vincent Tinto's Student integration model of attrition. Retrieved September 15, 2021 from https://rb.gy/ksw2vx

Calaor, P. M., Cartin, A. M., Casia, A. M., Macahilas, R. G. \& at Rivarez, R. A. (2020). Epekto ng online learning mode sa aspektong sikolohikal ng mga mag-aaral sa kursong Accountancy sa Pamantasang Teknolohikal ng Rizal - Pasig Campus. Retrieved September 15, 2021 from https://rb.gy/fbilm8

Carlo, B. (2019). Kahulugan at kahalagahan ng edukasyon. Retrieved September 15, 2021 mula sa https://www.pinoynewbie.com/kahulugan-kahalagahan-ng-edukasyon/

Ceka, A., \& Murati, R. (2016). The role of parents in the education of children. Journal of Education and Practice, 7 (5), 2222-288X.

CHEd Memo. 04, s.2020. (2 September 2020). Guidelines on the implementation of flexible learning. Retrieved August 10, 2021 from https://rb.gy/wfkc3f

Dhawan, S. (2020). Online learning: A panacea in the time of Covid-19 crisis. Journal of Technology System, 49(1), 5-22.

Del Castillo, F. (2021). COVID-19 impact on learners: challenges and responses, Journal of Public Health, 43(3), 539-540.

Ducanes, G. M. \& Tan, E. A. (2020). Pandemic drop-outs bring school population below 2011-2012 levels. Retrieved August 21, 2021 from https://rb.gy/rqatic

Endraca, G., (2020). Metro Manila: Epekto ng online class sa mga estudyante. Retrieved September 15, 2020 from https://www.radyolaverdad.com/pinagaaralan-ng-ched/

Franche, D. (2020). DepEd, 'di handa sa virtual classes, pagbubukas ng klase suspindihin - solon. Philstar.com. Retrieved September 15, 2021 from https://rb.gy/uzcubg

Gibbs, G. (2012). Analyzing qualitative data: The SAGE qualitative research kit. London: SAGE Publication.

Gotinga, J. (2020). More than 2 million 'dropouts' as schools adapt to pandemic. Retrieved August 20, 2021 from https://rb.gy/yelpix

Gunigundo, M., (2020). Online learning sa new normal ng edukasyon. Retrieved September 10, 2021 from https://rb.gy/xqscmi

K12academics.com. (2021). Theories and reasons for high school dropouts. Retrieved Setyembre 10, 2021, from https://rb.gy/umkyzl

Kühl, T., \& Eitel, A. (2016). Effects of disfluency on cognitive and metacognitive processes and outcomes Metacognition Learning. Springer Science+Business Media, 11, 1-13. DOI: https://doi.org/10.1007/s11409-016-9154-x

Labramonte, L. (2021). Kalagayan ng edukasyon sa panahon ng pandemya sa danas ng mga guro. Retrieved 
September 20, 2021 from https://rb.gy/pdyzzs

Li, C. \& Lalani, F. (2020). The COVID-19 pandemic has changed education forever: This is how. Retrieved September 10, 2021, from https://rb.gy/bdqz39

Magsambol, B. (2020). 44,000 college students won't enroll during pandemic. Retrieved August 15, 2021 from https://www.rappler.com/nation/college-students-wont-enroll-pandemic-pasuc

McCombes, S. (2019). What and how to use a case study. Retrieved September 20, 2021 from https://www.scribbr.com/methodology/case-study/

Moore, A. (2017). Dropped out: factors that cause students to leave before graduation. Unpublished doctoral dissertation. Carson-Newman University, Tennessee.

Nardo, M. B. (2017). Modular instruction enhances learner autonomy. American Journal of Educational Research, 5(10).

Officialgazette.gov.ph. (nd). The 1987 Constitution of he Republic of the Philippines - article XIV. Retrieved September 20, 2021 from https://rb.gy/4cbso9

Orion, H. C. Jr., Forosuelo, E. D. \& Cavalida, J. M. (2014). Factors affecting students' decision to drop out of school. Slongan, 2 (1). Retrieved September 15, 2021 from https://rb.gy/vsutnq

Pe Dangle, Y. R. \& Sumaoang, J. D. (2020, November 27-29). The implementation of modular distance learning in the Philippine secondary public schools. Paper presented at the $3^{\text {rd }}$ International Conference on Advance Research in Teaching and Education, Dublin, Republic of Ireland.

Nieto, S. M. (2020). Edukasyon sa kasagsagan ng pandemya. Retrieved August 20, 2021, form https://www.remate.ph/edukasyon-sa-kasagsagan-ng-pandemya

Ruhalahti, S., Lehto, T., Saarinen, S., \& Katto, L. (2021). Identifying Higher Education first-year students' reported studying experiences studying during the pandemic. European Journal of Education Studies, 8(8).

Vercide, J., (2020). Online classes epekibo nga ba para matuloy ang pasukan. Retrieved August 10, 2021, form https://thelookout.com.ph/article/online-classes-epektibo-nga-ba-para-matuloy-ang-pasukan

Vonderwell, S. (2003). An examination of asynchronous communication experiences and perspectives of students in an online course: a case study. The Internet and Higher Education, 6(1) 77-90.

Willging, P. A., \& Johnson, S. D. (2009). Factors that influence students' decision to drop out of online courses. Journal of Asynchronous Learning Networks, 13(3). 